\title{
Nonlinear Optimization of GM(1,1) Model Based on Multi-parameter Background Value
}

\author{
Tangsen Zhan and Hongyan Xu \\ School of Information Engineering, Jingdezhen Ceramic Institute, \\ Jingdezhen, Jiangxi, China \\ ztangsendyahoo.com.cn
}

\begin{abstract}
By studying the existing algorithms for background value in $\operatorname{GM}(1,1)$, a nonlinear optimization model of $\operatorname{GM}(1,1)$ based on multi-parameter background value is given. The paper uses the invertible matrix of the parameter to optimize and estimate the parameters $\hat{a}$; in addition, the parameter estimate $\hat{a}$ obtained from the multi-parameter background value has higher prediction accuracy, thus overcoming the restriction on the prediction based on the fixed average background value in other literatures. the simulated values obtained by the optimized model $(\operatorname{NOGM}(1,1))$ are more precise, and the maximum error is reduced by $15 \%$. The nonlinear optimization model of $\operatorname{GM}(1,1)$ based on multi-parameter background value provides algorithms for further study of $\mathrm{GM}(1,1)$ model.
\end{abstract}

Keywords: background value, parameter, nonlinear optimization, ratio.

\section{Introduction}

The grey model $\mathrm{GM}(1,1)$ is a forecast model in grey system. It is important to the application of grey system theory. Successful examples of using $\operatorname{GM}(1,1)$ for prediction can be found in many fields [1-2]. Much research has been done on this model, and a number of methods have been proposed to improve it [3-9]. Fundamentally, the effect of the change of the background value on the fitted value has been analyzed. However, when solving $\operatorname{GM}(1,1)$, the area is partitioned and the background value is obtained through approximate iteration, or the solution can be obtained through a parameter expression derived from a special background value model.

As for the background value, the $(1-\delta: \delta)$ ratio established between the background value and the two endpoint prototype values restricts the expression and estimation of grey differential equation, which causes the estimated parameters to produce a greater prediction error. The model solution is probably imprecise. This paper discusses the $\mathrm{GM}(1,1)$ model of the background value with two or more parameters obtained through the extension of the background value, the nonlinear optimization of the analog value with parameters called the nonlinear optimization model of $\operatorname{GM}(1,1)$ based on the background value with multi-parameters $(\operatorname{NOGM}(1,1))$. 


\section{The Nonlinear Optimization Model of GM(1,1) Based on the Multi-parameter Background Value}

\subsection{The Grey Model GM(1,1) of Background Value with Two Parameters}

Denote the original sequence by $X^{(0)}=\left(X_{(1)}^{(0)}, X_{(2)}^{(0)}, \ldots, X_{(N)}^{(0)}\right)$, calculate an AGO $X_{(k)}^{(1)}=\sum_{i=1}^{k} X_{(i)}^{(0)}$, get the new sequence $X^{(1)}=\left(X_{(1)}^{(1)}, X_{(2)}^{(1)}, \ldots, X_{(N)}^{(1)}\right)$, albinism differential form of $\operatorname{GM}(1,1)$ is

$$
\frac{d X^{(1)}}{d t}+a X^{(1)}=b
$$

Difference form of $\operatorname{GM}(1,1)$ is

$$
X_{(k)}^{(0)}+a^{*} z_{(k)}^{(1)}=b(\mathrm{k}=2,3,4, \ldots, \mathrm{N})
$$

There is $z_{(k+1)}^{(1)}=\mathrm{r}_{\mathrm{k}} * X_{(k)}^{(1)}+s_{k} * X_{(k+1)}^{(1)}$, solving (2) and getting parameter

$$
\hat{a}=[a, b]^{T}=\left(B^{T} B\right)^{-1} B^{T} Y_{N}
$$

Where $Y_{N}=\left(X_{(2)}^{(0)}, X_{(3)}^{(0)}, \ldots, X_{(N)}^{(0)}\right)$,

$$
B=\left[\begin{array}{llll}
1 & 1 & \ldots & 1 \\
-z_{(2)}^{1} & -z_{(2)}^{1} & \ldots & -z_{(N)}^{1}
\end{array}\right]^{T} \text {, the model of the background value } z_{(k+1)}^{(1)}
$$

with parameters $r_{k}$ and $s_{k}$ is obtained, and it is called the grey model GM(1,1) of the background value with two parameters.

\subsection{The Nonlinear Optimization Model of GM(1,1) for the Multi-parameter} Background Value

In order to achieve parameter estimation based on the multi-parameter background value, inverse matrix must be directly expressed by easy other matrix. The following is the process to get the parameter estimate through invertible matrix.

Theorem 1. If $\mathrm{F}=\sum_{k=2}^{N}\left(z_{(k)}^{(1)}\right)^{2}$, and $\mathrm{C}=\sum_{k=2}^{n} z_{(k)}^{(1)}$, 
$\left(B^{T} B\right)^{-1}=\frac{1}{(N-1) F-C^{2}}\left[\begin{array}{cc}N-1 & C \\ C & F\end{array}\right]$

Proof: Because $B^{T} B=\left[\begin{array}{ll}\sum_{k=2}^{N}\left(z_{(k)}^{(1)}\right)^{2} & -\sum_{k=2}^{N} z_{(k)}^{(1)} \\ -\sum_{k=2}^{N} z_{(k)}^{(1)} & N-1\end{array}\right]=\left[\begin{array}{cc}F & -C \\ -C & N-1\end{array}\right]$,

$\left(B^{T} B\right)^{-1}=\frac{\left(B^{T} B\right)^{*}}{\left|B^{T} B\right|}=\frac{1}{(N-1) F-C^{2}}\left[\begin{array}{cc}N-1 & C \\ C & F\end{array}\right]$.

Theorem 2. If $\mathrm{D}=\sum_{k=2}^{N} X_{(k)}^{(0)}$ and $\mathrm{E}=\sum_{k=2}^{N} X_{(k)}^{(0)} \cdot z_{(k)}^{(1)}, \quad B^{T} Y_{N}=\left[\begin{array}{l}-E \\ D\end{array}\right]$

Theorem 2 is easily established.

Derived from Theorem 1and Theorem 2 is equation (3), the parameter estimate $\hat{a}=[a, b]^{T}=\left(B^{T} B\right)^{-1} B^{T} Y_{N}$, where

$$
a=\frac{C^{*} D-(N-1) E}{(N-1)^{*} F-C^{2}}, \quad b=\frac{D^{*} F-C^{*} E}{(N-1) F-C^{2}}
$$

Substituting $a$ and $\mathrm{b}$ in the discrete responsive type

$$
\hat{X}_{(k+1)}^{(1)}=\left(X_{(1)}^{(0)}-\frac{b}{a}\right) * e^{-a^{* k}}+\frac{b}{a}
$$

Because matrix B and estimation parameter $\hat{a}$ include parameters $r_{k}$ and $s_{k}$, parameters $a$ and $\mathrm{b}$ cannot be directly calculated by (3), but will be calculated by the nonlinear optimization model as follows:

Step 1: Get $a$ and b with parameters $r_{k}$ and $s_{k}$ and the expression of the fitted value.

Get the fitted value $\hat{X}_{(k+1)}^{(0)}$ of the original data $X_{(k+1)}^{(0)}$ from form (5), and $\hat{X}_{(k+1)}^{(0)}=\hat{X}_{(k+1)}^{(1)}-\hat{X}_{(k)}^{(1)} \quad(k=0,1, \ldots, \mathrm{N}-1), \quad \hat{X}_{(1)}^{(0)}=X_{(1)}^{(0)}$ 
Step 2: Get and solve the expression of the minimum object function:

$$
\min f\left(r_{i}, s_{i}\right)=\sum_{k=2}^{N}\left(\hat{X}_{(k)}^{(0)}-X_{(k)}^{(0)}\right)^{2}
$$

Use MATLAB to program and calculate $r_{k}$ and $s_{k}$.

Step 3: Substitute $r$ and $s$ in (3) to calculate $a$ and b, substitute $r_{k}$ and $s_{k}$ in (4) to calculate the fitted value $\hat{X}_{(k+1)}^{(1)}$, and get the error values.

\section{Application of the Nonlinear Optimization Model of GM(1,1) for the Background Value with Parameters}

Use the GDPs of china from 1991 to 2003 to set background values with two parameters $r$ and $s$ in $\operatorname{NOGM}(1,1)$, and get $r=0.0267, s=0.8945$, but $r+s \neq 1$ through (3), (4), and (5). The results show that the model that expresses the background value better is not based on the $(1-\delta: \delta)$ ratio between the two endpoint values. The values simulated in the $\operatorname{NOGM}(1,1)$ are compared with those simulated in $\operatorname{NGM}(1,1)[7]$ in the following table 1 .

Table 1. Comparison between values simulated in $\operatorname{NOGM}(1,1)$ and those in $\operatorname{NGM}(1,1)$

\begin{tabular}{|c|c|c|c|c|c|}
\hline \multirow[t]{2}{*}{ year } & \multirow[t]{2}{*}{$\begin{array}{c}\text { Original } \\
\text { data }\end{array}$} & \multirow[t]{2}{*}{$\begin{array}{l}\text { Simulated } \\
\text { data in } \\
\operatorname{DGM}(1,1)\end{array}$} & \multirow{2}{*}{$\begin{array}{c}\text { Simulated } \\
\text { error in } \\
\operatorname{DGM}(1,1) \\
\% \\
\end{array}$} & $\begin{array}{l}\text { Simulated data in } \\
\operatorname{NOGM}(1,1) \text { with } \\
\text { two parameters }\end{array}$ & $\begin{array}{c}\text { Simulated error in } \\
\operatorname{NOGM}(1,1) \text { with two } \\
\text { parameters \% }\end{array}$ \\
\hline & & & & $a=-0.1040, b=34468$ & \\
\hline 1991 & 21617.8 & 21617.8 & 0 & 21617.8 & 0 \\
\hline 1992 & 26638.1 & 40500 & 52.04 & 36548 & 37.21 \\
\hline 1993 & 34634.4 & 44750 & 29.22 & 42754 & 23.4437 \\
\hline 1994 & 46759.4 & 49450 & 5.76 & 47419 & 1.41063 \\
\hline 1995 & 58478.1 & 54640 & 6.56 & 52594 & 10.0621 \\
\hline 1996 & 67884.6 & 60380 & 11.05 & 58332 & 14.0718 \\
\hline 1997 & 74462.6 & 66720 & 10.4 & 64697 & 13.1148 \\
\hline 1998 & 78345.2 & 73720 & 5.9 & 71756 & 8.4107 \\
\hline 1999 & 82067.5 & 81460 & 0.73 & 79586 & 3.02373 \\
\hline 2000 & 89468.1 & 90020 & 0.61 & 88269 & 1.34025 \\
\hline 2001 & 97314.8 & 99470 & 2.21 & 97901 & 0.602375 \\
\hline 2002 & 105172.3 & 109910 & 4.51 & 108580 & 3.24011 \\
\hline 2003 & 117251.9 & 121450 & 3.58 & 120430 & 2.71049 \\
\hline Average & & & 10.1977 & & 9.167 \\
\hline
\end{tabular}




\section{Conclusion}

GM(1,1) model has its adaptation range[6]. By optimizing and making background values parametric, the paper uses the invertible matrix of the parameter to optimize and estimate the parameters $\hat{a}$, the improved model overcomes the dependence of the background value on the $(1-\delta: \delta)$ ratio between the two endpoint values in other literatures. It provides a better prediction model for the conversion from a continual form to a discrete form. The examples indicate that the simulated value obtained by the model $\operatorname{NOGM}(1,1)$ is more precise, and the maximum error is reduced by $15 \%$. However, because the simulated value is expressed by an exponential function, the simulating error is bigger at some points. Then, how to reduce the overall error will be a concern of the future study.

Acknowledgements. Supported by the National Natural Science Foundation of China under Grant No.61066003.

\section{References}

[1] Shui, N., Qin, Y.: On some theoretical problems of GM(1,1) model of grey systems. Systems Engineering-Theory \& Practice 18(4), 59-63 (1998)

[2] Liu, S., Guo, T., Dang, Y.: Grey System Theory and Its Applica-tion. Science Press, Beijing (2004)

[3] Tan, G.-J.: The structure method and application of background value in grey system GM(1,1) model (I). Systems Engineering-Theory \& Practice 4(4), 98-103 (2004)

[4] Wang, Y., Liu, G.: A step by step optimum direct modeling method of $\operatorname{GM}(1,1)$. Systems Engineering-Theory \& Practice 9(6), 99-104 (2000)

[5] Xie, N., Liu, S.: Discrete $\operatorname{GM}(1,1)$ and mechanism of grey forecasting model. Systems Engineering-Theory \& Practice 1(3), 93-99 (2005)

[6] Xu, H., Chen, Y., Fei, Z.: Discussion of the region suitable for $\operatorname{GM}(1,1)$ and its improving model. Mathematics in Practice and Theory 11(4), 58-63 (2008)

[7] Fei, Z., Xu, H., Jiang, Z.: Theoretical defect of GM(1,1) model and its optimum analysis based on time response function. Mathematics in Practice and Theory 5(10), 214-219 (2009)

[8] Liu, N., Sun, W., Yao, H.: Research on improving the fitting and prediction precision of the GM $(1,1)$ Model. Mathematics in Practice and Theory 4(8), 33-39 (2008)

[9] Chen, S., Li, Z., Zhou, S.: Application of non-equal interval $\operatorname{GM}(1,1)$ model in oil monitoring of internal combustion engine. Journal of Central South University of Technology 12(6), 705-708 (2005) 